\title{
Eosinophilic oesophagitis: recent advances and practical management
}

\author{
Stephen Attwood (D) , ${ }^{1}$ Jenny Epstein ${ }^{2}$
}

${ }^{1}$ Health Services Research, Durham University, Durham, UK ${ }^{2}$ Paediatric Gastroenterology, Chelsea and Westminster Hospital NHS Foundation Trust, London, UK

\section{Correspondence to} Jenny Epstein, Chelsea and Westminster Hospital NHS Foundation Trust, London SW10 9NH, UK; jenny.epstein@ chelwest.nhs.uk

Received 15 June 2020 Revised 14 August 2020 Accepted 15 August 2020 Published Online First

7 September 2020

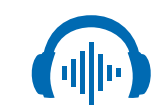

Listen to Podcast gutjnl.bmj.com

\section{Check for updates}

(C) Author(s) (or their employer(s)) 2021. No commercial re-use. See rights and permissions. Published by BMJ.

To cite: Attwood S, Epstein J. Frontline Gastroenterology 2021;12:644-649.

\begin{abstract}
Eosinophilic oesophagitis (EoE) is a disease identified just over 30 years ago. The main symptom is dysphagia. EoE is initially inflammatory and progresses to fibrosis. There are differences in clinical presentation between young children and adults. Diagnosis is by endoscopy and six biopsies at varying positions of the oesophageal lining. Blood tests are of no diagnostic value as the condition is mediated by $\operatorname{lgG}_{4}$ local mucosal pathology. Endoscopic signs are distinct from those of gastro-oesophageal reflux. Histological signs of EoE are $>15$ eosinophils/high-power field on a background of hyperplastic mucosa. Options of therapy include diet restriction, proton pump inhibitors therapy and topical steroids but there is a dearth of randomised control trials to define the optimum approach. The only licenced therapy for EoE is budesonide orodispersible tablet, a specific formulation for oesophageal topical steroid therapy. EoE is the most common cause of spontaneous perforation in the oesophagus. Stricture formation occurs in up to $10 \%$ and may require therapeutic dilatation.
\end{abstract}

\section{INTRODUCTION}

The diagnosis that every gastroenterologist needs to know about for the 2000s is eosinophilic oesophagitis (EoE). Since its initial description in $1993^{1}$ and its eventual accreditation with International Classification of Diseases-10 code (K.20.0) in $2011,{ }^{2}$ EoE has become the second most common oesophageal disease and arguably the most common cause of dysphagia in young people.

Eosinophilic inflammation may arise anywhere in the gastrointestinal tract, although the most common and best categorised disease site is that seen in EoE. In health, eosinophils have a sentinel function and are present in small numbers throughout the gut except the oesophagus. They protect against helminthic and other infections. In disease, symptoms are diverse and depend on the site of inflammation, both in terms of position from oesophagus to colon, and position within the layers of the wall (mucosal causing diarrhoea; serosal causing ascites).

The symptoms in EoE are caused by difficulties during passage of food through the oesophagus. Patients feel food stick after swallowing, or a chest discomfortcentral, upper, mid or lower. They may use the terms 'indigestion' or 'heartburn' but not realise they differ in nature from discomfort felt by people who suffer acid reflux. The symptoms occur during or immediately after swallowing, during a meal. This contrasts with reflux which occurs postprandially. The patient may describe regurgitation, if swallowed fluids (saliva or drinks) cannot pass the stuck food bolus. This does not taste acidic, nor of gastric content and is quite distinct from the regurgitation in gastro-oesophageal reflux disease (GORD).

The condition is inflammatory, with a predominant eosinophilic inflammation, and progressively fibrotic. ${ }^{3}$ Both the primary inflammation and fibrosis contribute to the relative obstruction to passage of solid foods, resulting in dysphagia, food bolus obstruction, odynophagia and awareness of slow food passage. EoE sufferers find they are last to finish a meal, use large amounts of liquid to wash food down, or suffer the indignity of food bolus obstruction and regurgitate all subsequent swallowed saliva, fluids or solids until the bolus has shifted. When this occurs while eating in public this creates added stress.

EoE is a disease of younger peoplechildren and young adults. The manifestation of EoE symptoms in children may differ from that in adults. Parental interpretation of their child's eating difficulty may be relayed as regurgitation or food refusal, both possibly due to dysphagia and temporary bolus obstructions. A young child approaching solid food for 
the first time may not know the circumstance of normal swallowing or eating food of normal solid consistency. Dysphagia and behavioural difficulties associated with eating in young children may be expressed differently to those who acquire the symptom in adulthood. ${ }^{4} \mathrm{EoE}$ in children may also arise on a background of other food intolerances and allergies. Infants and toddlers tend to present with non-specific symptoms such as feeding difficulties, failure to thrive, regurgitation and discomfort; while in mid-school-age children abdominal pain and vomiting become more prominent. In older children the presentation of EoE changes due to progression of fibrosis, with the adult phenotype of dysphagia and food impaction. ${ }^{4}$

Children and their carers adapt to their symptoms, with unconscious behavioural modifications to food choices and eating patterns. Clinicians need to maintain suspicion of EoE; taking focused history with specific questions about eating adaptations, excessive water drinking, solid texture avoidance and slow eating.

\section{Epidemiology}

When first described EoE was regarded as rare. In the past decade there has been rapid rise in prevalence throughout the western world, with mean estimates at 15/100 000 before 2007 and 63/100 000 since 2017.5

This puts the condition on par with inflammatory bowel disease. The peak incidence is seen in young adults (third and fourth decade) but recognised in all ages. It is three times commoner in males and associated with other atopic diseases such as allergic asthma, rhinitis and eczema.

The complication of food bolus obstruction is the most frequent emergency presentation of EoE, occurring in $20 \%$ of sufferers. EoE is the most common cause of acute food bolus obstruction seen in emergency departments, ${ }^{67}$ the most common cause of spontaneous oesophageal perforation with presentation and character significantly different from that seen in Boerhaave syndrome (see Complications : Perforation).

There is seasonality in presentation of EoE, more new diagnoses being made in spring and summer. Food bolus impactions occur more frequently in summer and autumn. This implies an environmental trigger, possibly airborne but it could equally relate to seasonal availability of foods.

\section{Diagnosis}

The only avenue to a diagnosis of EoE is to assess the histology of the oesophageal mucosa. That requires an endoscopy, which itself may reveal characteristic signs of EoE such as linear furrows, rings or trachealisation, microabscesses, mucosal fragility, oedema, stricture or narrow bore oesophagus. ${ }^{8}$ In $10 \%-20 \%$ of adults, and more in children, the oesophagus may appear normal. Regardless of the presence or absence of indicative signs of EoE every patient with dysphagia, or atypical childhood presentation of significant oesophageal symptoms requires biopsy. As inflammation is patchy biopsies must be multiple and multi-focal. Studies have identified that six samples are needed, either two each from lower, mid and upper oesophagus, or three from each half, whichever is most convenient. ${ }^{910}$

The presence of dense infiltration of eosinophils within the oesophageal epithelium with a peak concentration of $>15$ eosinophils/high-power field (hpf) is the distinguishing feature of EoE from other diseases. Although GORD may have a few eosinophils present these are usually less than 5/hpf. Both GORD and EoE show histological features of hyperplastic mucosa with increased basal zone thickness and increased papillary height. In both some degree of increased cellular spacing may occur. In GORD the inflammation is usually restricted to the lower oesophagus and worst at the GE junction, whereas in EoE the inflammation may be patchy, scattered throughout the oesophagus. A common error is to assume that biopsies near the oesophago-gastric junction showing high density of eosinophils relate to GORD but this more likely to represent EoE because high density eosinophilia is rare in GORD.

It is important to understand that in adulthood EoE and GORD are separate conditions. They present with different gender and atopy background. Symptoms differ in character and come on at different timesduring meals for EoE but after meals or at night for GORD. They look completely different endoscopically and they have a different range of histological appearances. They have different causes with acid reflux inducing GORD but antigen driven immune reactivity in EoE. As GORD is common, being seen in up to $30 \%$ of our adult population it is possible for both to coexist.

Some diseases such as achalasia, Chagas disease and hypereosinophilic syndromes, may possibly have an oesophageal eosinophil infiltrate but these are much less frequent, and most of these patients do not have $>15$ eosinophils/hpf.

There are no blood tests for diagnosis of EoE. The underlying pathology in EoE is an $\operatorname{IgG}_{4}$ mucosal reaction $^{11}$ in which there is little systemic involvement. In a small proportion of patients, peripheral eosinophil counts may be elevated but this is non-specific, and insensitive. Tests of $\operatorname{IgG}, \operatorname{IgE}$ or IgA levels are not helpful. If allergy tests are used they contribute to the management of coexistent asthma or eczema but do not contribute to diagnosis or management of EoE. Tests of skin reactivity are not correlated with oesophageal reactivity ${ }^{12}$ in adults, and poorly correlated in children. Although it is possible to use the Oesophageal String test ${ }^{13}$ this is currently not available as a diagnostic indicator. Work is underway to assess its value in monitoring response to therapy.

In young children overlap between GORD and EoE is relatively common; GORD in infants and toddlers 
being driven by underlying milk allergy, yielding borderline mucosal eosinophil counts and often responding to the exclusion of milk from the diet. This is a frequent source of diagnostic uncertainty, not least because treatments common to both infantile GORD and EoE, namely milk-free diet and proton pump inhibitors (PPi) medication are used empirically before endoscopy.

The main issue for most patients with EoE is a reduction in quality of life, with social restrictions, difficulties with eating and dietary problems. These are the factors that drive the majority of patients to seek medical help.

\section{Therapy}

No published guideline gives directions about the best therapeutic option in EoE due to a distinct lack of comparative randomised controlled trials. No one, simple, algorithm is likely to fit all patients. The following arguments represent the experience of the authors and will understandably differ from others.

Decisions on treatment of EoE require assessment of the impact the disease has on the patient's life and an understanding of the patient's own philosophy regarding treatment options. During the initial assessment, symptom severity should be recorded. Symptom frequency varies from every meal and every day to only once or twice a week, or month. Symptom severity ranges from food bolus obstruction requiring emergency attendance to minor and intermittent dysphagia. Many patients adapt well by modifying their diet, mincing their food, liquidising their vegetables and surviving on soups, smoothies and liquids.

Clinicians should describe the treatment options alongside their impact on the patient's quality of life and likelihood of future normal eating.

Diet

If food antigens are responsible for oesophageal eosinophilic inflammation, then their exclusion should be a simple therapy. Unfortunately, most patients react to multiple food types. Currently there are no reliable methods of ascertaining which foods are responsible. Skin prick testing identifies allergens associated with $\mathrm{IgE}$ mediated atopies such as eczema and rhinitis but not the $\mathrm{IgG}_{4}$ EoE. Two practical approaches to diet are in use-a step up 2-4-6 food elimination and a step down 6-food elimination. Both require careful support by dietitians experienced in EoE. Both require follow-up and investigation to identify if initial symptom improvements are likely to provide a longlasting therapeutic strategy. There is poor correlation of symptoms and histological improvement in EoE, partly related to placebo type responses, and partly to the influence of dietary behaviours and food avoidance by the patients. ${ }^{14-16}$

The foods that stick during swallowing are usually not the foods that are the source of pro-inflammatory drive. Over the past decade the most common approach has been the elimination of multiple foodsmilk, wheat, eggs, soya, nuts and fish. Often called the 6-food elimination diet this is a misnomer as the number of food types excluded in studies is often more, often excluding legumes. The range of success is $50 \%-75 \%$ of patients after 12 weeks, requiring both symptom check and endoscopy with biopsy to ascertain true remission. ${ }^{14}{ }^{15}{ }^{17}$ It is difficult to maintain a diet excluding all these food types, less than $10 \%$ of patients sustaining 6 -food elimination for 12 months. ${ }^{14} 15$

A simpler approach, the 2-4-6 step up diet is growing in popularity. Excluding milk and wheat is successful in $40 \%$ of patients and this is cheap, using a single follow-up endoscopy and practical to sustain. ${ }^{18}$ The decision regarding those who fail the 2-food elimination is more complex, as stepping up to four and then six foods introduces the difficulties of multiple endoscopies and difficulties in sustaining the diet. The decision on whether a patient persists with a dietary approach or switches to a drug therapy is an individual one that requires counselling.

Dietary therapies are equally efficacious in children and adults but used more in paediatric than adult settings. This may be driven by clinicians' and carers' reluctance to use steroids in children and, especially in younger children, the ability to impose restricted diets. The same foods are implicated in paediatric and adult EoE. There is evidence that allergy testing plays a slightly more useful role in children than adults in guiding the selection of diet. This may reflect the gradual switch over time of EoE from IgE-mediated to $\mathrm{IgG}_{4}$-mediated disease, hence the increased prevalence of IgE-based testing in childhood. Milk remains the most common food trigger and exclusion of milk should always be part of the initial dietary approach.

\section{Drug therapy: PPi}

Despite the lack of acid reflux in the aetiology of EoE there is a symptom response in $30 \%-60 \%{ }^{19}$ of patients in observational and uncontrolled studies, when double dose PPi (20 mg omeprazole two times per day or equivalent) are used for 6 weeks. Prospective studies of their use in routine practice are less optimistic. ${ }^{20}$ Outcomes of PPi in children are also less than those seen in adults. ${ }^{21}$ Due to their low cost and availability most recommend starting therapy with a $\mathrm{PPi}$, checked with endoscopy and a standard severity questionnaire to ensure objectivity to the assessment. If GORD coexists with EoE-which may occur in $10 \%-20 \%$ of patients-then PPi may have benefit for the reflux symptoms of heartburn.

\section{Drug therapy: topical steroid}

The most effective specific therapy for EoE is topical steroid. ${ }^{22}{ }^{23}$ Until the introduction of special oesophageal formulations their use was restricted by the 
complexity of administering formulations intended for bronchial asthma. This caused confusion in administration, dosage and clear patient information. Fluticasone spray $(250 \mu \mathrm{g}$ four puffs two times per day, last thing at night and after breakfast with no mouth washing, food or fluids for as long as possible) is suitable for adults, or a viscous solution of oral budesonide, ${ }^{23}$ variably formulated in hospital pharmacies and the previous standard of therapy in children, are effective but both lack consistency in delivery and formulations. This has driven the need for a dedicated formulation now available as an orodispersible budesonide tablet, which can be placed on the tongue and allowed to dissolve in saliva passing gradually into the oesophagus. In placebo-controlled trials, this is highly effective in symptom resolution and histological remission (85\% at 12 weeks). ${ }^{22}$ Symptom remission improves with longer duration of therapy and is a feature of the time it takes for fibrosis in EoE pathophysiology to normalise. Fibrosis takes a long time to develop and in some a long time to resolve. There may be patients who require a therapeutic dilatation. They must have continuation of topical steroid as their maintenance therapy. ${ }^{24}$ Safety of long-term budesonide for GI complaints is well established and the occasional oral thrush easily managed by oral nystatin suspension for 10 days, without cessation of therapeutic topical steroids.

\section{General considerations in choosing first line therapy}

In patients with severe (daily) symptoms, history of food bolus obstruction or those who have already had a trial of PPi, topical steroids may be the first line therapy. There is no value in using systemic steroids as they do not produce a greater concentration of steroid at the site of action (the oesophageal epithelium) and they have severe untoward side effects when used for long periods. ${ }^{21}$ Topical steroids need to be used for 3 months in the first instance and thereafter in maintenance therapy or in repeated bolus courses of 3 months. Work is on-going to define which of these approaches is appropriate.

With moderate symptoms, mild symptoms or occasional self-resolving bolus obstruction first use diet or PPi. In practice many patients have already tried PPi. The balance of using a cheap but unlicenced drug in the setting of needing more endoscopic checks must be offset against the costs of a licenced effective therapy. Dietary therapy is suited to well-motivated patients in whom there is a likelihood that they will comply with their diet in the long term. The lower the number of foods to avoid the easier that is to achieve. In children concerns around growth and weight gain can complicate instigation of restricted diets although EoE is not associated with reduced final height attainment. Long term dietary restrictions are challenging to adhere to especially in adolescence. Decision making between dietary and drug treatment is therefore typically led by individual and family preferences rather than medical factors, since efficacy of the two approaches are comparable.

\section{Follow-up}

Guidelines required endoscopy and follow-up to assess both symptoms and histology with all types of therapy. ${ }^{3} 1021$ The problem of needing to objectify disease response is because symptomatic responses are poor and have poor correlation with histological response, as is the case with PPi and dietary regimes. It might be that future guidelines could omit an endoscopy if $>90 \%$ have histological remission and $85 \%$ symptom improvement after 12 weeks with orodispersible budesonide, thereby reducing overall costs.

In paediatric practice there is an inherent challenge in the long-term management of EoE as the disease returns when the treatment is stopped. The safety of long-term swallowed steroid is not entirely established although evidence suggests it has a good safety profile in up to 2 years of continuous use in children. ${ }^{24}$ Monitoring for adrenal suppression is recommended.

Early fibrosis in EoE may be reversible, more so in childhood. If it becomes established it can lead to irreversible complications such as stricture, food bolus impaction and perforation, more commonly seen in adults.

\section{Complications}

\section{Food bolus obstruction}

EoE is now the most common cause of food bolus obstruction presenting to emergency departments. ${ }^{36}$ Although often treated by Ear Nose and Throat surgeons, there are good reasons for food bolus obstruction to be managed by gastroenterologists, as long as the airway is not compromised.

Management: Many patients tolerate temporary food bolus obstruction over a number of hours, and there is no clear definition of when emergency treatment is necessary. Most patients present to an Emergency Department within 4 hours, and much sooner if symptoms are distressing. The initial management is conservative, with reassurance, intravenous fluids and a clinical assessment to rule out perforation, excluding cervical surgical emphysema or 'crepitus'. If pain is predominant perform a chest X-ray to detect free air. Antispasmodics or muscle relaxants have no value. An urgent endoscopy should be arranged, with protection of the airway during bolus extraction. This may involve a general anaesthetic. At the first endoscopy make a full assessment of the cause, before or after careful extraction of the bolus. Biopsy above, below and at the site of obstruction should be performed. It is unfortunate that the lack of knowledge that EoE is the most common cause of bolus obstruction leads to lengthy delays of therapy and recurrence in many patients. ${ }^{25}$ The authors strongly advise biopsy at the index endoscopy to avoid diagnostic failures. 


\section{Perforation of the oesophagus}

EoE is the most common cause of spontaneous perforation in the oesophagus. ${ }^{3}$ The presence of severe chest pain with food bolus obstruction should alert the clinician to perforation. The patient is distressed with the pain and may have crepitus in the neck-surgical emphysema, crunchy to palpation. In contrast to the complete rupture of the lower oesophagus in Boerhaave's syndrome, the type of perforation in EoE is usually a partial tear, in multiple sites close together with extravasation of fluid and gas but not solids. There is usually no pleural involvement and although mediastinal air may be seen on a chest X-ray there is usually no pneumothorax.

Management of perforation ${ }^{3}$ is supportive with antibiotics, nil by mouth, a contrast CT to assess the degree of extravasation and then usually a conservative approach. Depending on the size of leak the radiological placement of a Naso-Gastric tube and intravenous $\mathrm{PPi}$, or if a moderate leak drainage of the mediastinal space using an endoscopically placed drainage tube, or a vacuum suction system as described by Wedemeyer et al. ${ }^{26}$ Care should be provided by a multi-disciplinary approach, including radiology, surgery and gastroenterology opinions and an awareness that few patients require surgical intervention or oesophageal resection.

\section{Stricture}

From recent publications it appears that the incidence of stricture in EoE is reducing. Some have a narrow bore oesophagus throughout its length which is hard to appreciate at routine endoscopy. In the authors' experience up to $10 \%$ may develop a stricture after the diagnosis is made, usually because of ineffective therapy. Progressive fibrosis occurs and its effects are most clearly assessed using the EndoFlip (Functional Luminal Planimetry) which measures the compliance and distensibility of the oesophagus ${ }^{27}$ which may help guide therapeutic dilatation of EoE in patients who fail medical therapies.

Management of stricture: In the first instance a trial of anti-inflammatory therapy is worthwhile but if it fails, or if the stricture is very tight then a therapeutic dilatation is indicated. Anti-inflammatory therapy should be continued to prevent recurrence. The details of dilatation in EoE are well described in the British Society of Gastroenterology Guideline for oesophageal dilatation therapy. ${ }^{28}$

\section{Psychosocial complications of therapy}

Patients left on elimination diets find them difficult to follow for social and practical reasons. Their quality of life becomes impeded despite improvement in EoE symptoms. Particularly in children and adolescents, the effects of the treatment by diet may be worse than the disease. ${ }^{29}$ Discuss the burden of therapy as well as the burden of disease when counselling patients and their families about the management of EoE.

\section{CONCLUSION}

With a structured approach to therapy, supported by standardised patient questionnaires to make a reproducible assessment of symptom severity and supported by endoscopic assessment of remission, therapy in EoE is usually successful. More than $90 \%$ of patients can be treated effectively in the authors' experience and failures are more often related to issues of compliance with the planned therapy than to failure of all three therapies completely. Although some biological agents are being developed that may directly interfere with the pathogenesis of eosinophil biology their expense and their potential side effects may give them only a limited role in EoE. In patients with multiple gastrointestinal eosinophil diseases, a rare circumstance, such new drugs may have special value. ${ }^{30}$

The patient organisation (EosNetwork https:// www.eosnetwork.org/), the European Eosinophilic Oesophagitis Society (Eureos https://eureos.online/ home-eureos.html) and the European Society for Paediatric Gastroenterology, Hepatology and Nutrition (ESPGHAN https://www.espghan.org/) have useful material on their websites to help guide decisions on diagnosis and management of EoE.

Contributors Both authors contributed directly to the development, writing and editing of this review.

Funding The authors have not declared a specific grant for this research from any funding agency in the public, commercial or not-for-profit sectors.

Competing interests SA is a paid speaker for the following companies related to the treatment of oesophageal diseases, Dr Falk Pharma, Medtronic Inc and research advisor to Dr Falk Pharma, AstraZeneca and EsoCap Inc. JE is a paid speaker and advisor to Dr Falk Pharma. Both authors are voluntary advisors to EosNetwork, Euroes and the BSG Guideline Development group, and JE an advisor to ESPGHAN guidelines.

Patient consent for publication Not required.

Provenance and peer review Commissioned; externally peer reviewed.

ORCID iD

Stephen Attwood http://orcid.org/0000-0002-5761-157X

\section{REFERENCES}

1 Attwood SE, Smyrk TC, Demeester TR, et al. Esophageal eosinophilia with dysphagia. A distinct clinicopathologic syndrome. Dig Dis Sci 1993;38:109-16.

2 CPT. International statistical classification of diseases and related health problems: ICD-10. Available: https://coder.aapc. com/icd-10-codes/K20.0\#: : text $=$ ICD $\% 2 D 10 \% 20$ code $\%$ 20K20.,Diseases $\% 20$ of $\% 20$ the $\% 20$ digestive $\% 20$ system $\% 20$ [Accessed 15 Jun 2020].

3 Liacouras CA, Furuta GT, Hirano I, et al. Eosinophilic esophagitis: updated consensus recommendations for children and adults. J Allergy Clin Immunol 2011;128:3-20.

4 Spergel JM, Brown-Whitehorn TF, Beausoleil JL, et al. 14 years of eosinophilic esophagitis: clinical features and prognosis. $J$ Pediatr Gastroenterol Nutr 2009;48:30-6.

5 Navarro P, Arias Ángel, Arias-González L, Arias A A-G, et al. Systematic review with meta-analysis: the growing incidence and prevalence of eosinophilic oesophagitis in children and adults in population-based studies. Aliment Pharmacol Ther 2019;49:1116-25. 
6 Ntuli Y, Bough I, Wilson M. Recognising eosinophilic oesophagitis as a cause of food bolus obstruction. Frontline Gastroenterol 2020;11:11-15.

7 Dellon ES, Kim HP, Sperry SLW, et al. A phenotypic analysis shows that eosinophilic esophagitis is a progressive fibrostenotic disease. Gastrointest Endosc 2014;79:577-85.

8 Hirano I, Moy N, Heckman MG, et al. Endoscopic assessment of the oesophageal features of eosinophilic oesophagitis: validation of a novel classification and grading system. Gut 2013;62:489-95.

9 Nielsen JA, Lager DJ, Lewin M, et al. The optimal number of biopsy fragments to establish a morphologic diagnosis of eosinophilic esophagitis. Am J Gastroenterol 2014;109:515-20.

10 Lucendo AJ, Molina-Infante J, Arias Ángel, et al. Guidelines on eosinophilic esophagitis: evidence-based statements and recommendations for diagnosis and management in children and adults. United European Gastroenterol J 2017;5:335-58.

11 Clayton F, Fang JC, Gleich GJ, et al. Eosinophilic esophagitis in adults is associated with IgG4 and not mediated by IgE. Gastroenterology 2014;147:602-9.

12 Philpott H, Nandurkar S, Royce SG, et al. Allergy tests do not predict food triggers in adult patients with eosinophilic oesophagitis. A comprehensive prospective study using five modalities. Aliment Pharmacol Ther 2016;44:223-33.

13 Furuta GT, Kagalwalla AF, Lee JJ, et al. The oesophageal string test: a novel, minimally invasive method measures mucosal inflammation in eosinophilic oesophagitis. Gut 2013;62:1395405.

14 Gonsalves N, Yang G-Y, Doerfler B, et al. Elimination diet effectively treats eosinophilic esophagitis in adults; food reintroduction identifies causative factors. Gastroenterology 2012;142:1451-9.

15 Kagalwalla AF, Sentongo TA, Ritz S, et al. Effect of sixfood elimination diet on clinical and histologic outcomes in eosinophilic esophagitis. Clin Gastroenterol Hepatol 2006;4:1097-102.

16 Kelly KJ, Lazenby AJ, Rowe PC, et al. Eosinophilic esophagitis attributed to gastroesophageal reflux: improvement with an amino acid-based formula. Gastroenterology 1995;109:150312.

17 Arias A, González-Cervera J, Tenias JM, et al. Efficacy of dietary interventions for inducing histologic remission in patients with eosinophilic esophagitis: a systematic review and meta-analysis. Gastroenterology 2014;146:1639-48.

18 Molina-Infante J, Arias Ángel, Alcedo J, et al. Step-up empiric elimination diet for pediatric and adult eosinophilic esophagitis: the 2-4-6 study. J Allergy Clin Immunol 2018;141:1365-72.
19 Lucendo AJ, Arias Ángel, Molina-Infante J. Efficacy of proton pump inhibitor drugs for inducing clinical and histologic remission in patients with symptomatic esophageal eosinophilia: a systematic review and meta-analysis. Clin Gastroenterol Hepatol 2016;14:13-22.

20 Philpott H, Nandurkar S, Royce SG, et al. A prospective open clinical trial of a proton pump inhibitor, elimination diet and/or budesonide for eosinophilic oesophagitis. Aliment Pharmacol Ther 2016;43:985-93.

21 Hoofien A, Dias JA, Malamisura M, et al. Pediatric eosinophilic esophagitis: results of the European retrospective pediatric eosinophilic esophagitis registry (RetroPEER). $J$ Pediatr Gastroenterol Nutr 2019;68:552-8.

22 Lucendo AJ, Miehlke S, Schlag C, et al. Efficacy of budesonide orodispersible tablets as induction therapy for eosinophilic esophagitis in a randomized placebo-controlled trial. Gastroenterology 2019;157:74-86.

23 Gupta SK, Vitanza JM, Collins MH. Efficacy and safety of oral budesonide suspension in pediatric patients with eosinophilic esophagitis. Clin Gastroenterol Hepatol $2015 ; 13: 66-76$

24 Andreae DA, Hanna MG, Magid MS, et al. Swallowed fluticasone propionate is an effective long-term maintenance therapy for children with eosinophilic esophagitis. Am J Gastroenterol 2016;111:1187-97.

25 Tourlamain G, Garcia-Puig R, Gutiérrez-Junquera C, et al. Differences in management of eosinophilic esophagitis in Europe: an assessment of current practice. J Pediatr Gastroenterol Nutr 2020;71:83-90.

26 Wedemeyer J, Brangewitz M, Kubicka S, et al. Management of major postsurgical gastroesophageal intrathoracic leaks with an endoscopic vacuum-assisted closure system. Gastrointest Endosc 2010;71:382-6.

27 Kwiatek MA, Hirano I, Kahrilas PJ, et al. Mechanical properties of the esophagus in eosinophilic esophagitis. Gastroenterology 2011;140:82-90.

28 Sami SS, Haboubi HN, Ang Y, et al. UK guidelines on oesophageal dilatation in clinical practice. Gut 2018;67:100023.

29 Harris RF, Menard-Katcher C, Atkins D, et al. Psychosocial dysfunction in children and adolescents with eosinophilic esophagitis. J Pediatr Gastroenterol Nutr 2013;57:500-5.

30 Rank MA, Sharaf RN, Furuta GT, et al. Technical review on the management of eosinophilic esophagitis: a report from the AGA Institute and the joint Task force on allergy-immunology practice parameters. Ann Allergy Asthma Immunol $2020 ; 124: 424-40$. 